\title{
Synchronized dual pulse gastric electrical stimulation improves gastric emptying and activates enteric glial cells via upregulation of GFAP and S100B with different courses of subdiaphragmatic vagotomy in rats
}

\author{
NIAN WANG, SHUANGNING SONG and JIE CHEN \\ Division of Gastroenterology, Union Hospital, Tongji Medical College, \\ Huazhong University of Science and Technology, Wuhan, Hubei 430022, P.R. China \\ Received January 25, 2016; Accepted February 16, 2017
}

DOI: $10.3892 / \mathrm{mmr} .2017 .6471$

\begin{abstract}
Previous research and clinical practice have indicated that damage to the vagal nerve may seriously affect gastrointestinal physiological movement behavior. The aim of the current study was to observe the change of gastric motility, as well as enteric glial cells (EGCs) in the stomach with different courses of vagal nerve transection in rats prior to and following synchronized dual pulse gastric electrical stimulation. The gastric emptying rates were measured to assess the gastric motility. The glial markers, containing calcium binding protein (S100B) and glial fibrillary acidic protein (GFAP), were detected by reverse transcription-quantitative polymerase chain reaction and double-labeling immunofluorescence analysis. Ultrastructural changes of EGCs were observed using transmission electron microscopy. Gastric emptying was delayed in the terminal vagotomy group, compared with the terminal control group. The effect of long-term synchronized dual pulse gastric electrical stimulation (SGES) was superior to short-term SGES in terminal groups. The expression levels of S100B/GFAP were markedly decreased in the terminal vagotomy group compared with the terminal control group. Following short-term or long-term SGES, S100B/GFAP gene and protein expression increased in terminal groups. However, long-term SGES was more effective than short-term SGES and the difference was statistically significant. Vagal nerve damage leads to gastric motility disorder and weakens the function of EGCs. Therefore, SGES may improve stomach movement behavior and restore the impaired EGCs. The underlying
\end{abstract}

Correspondence to: Dr Jie Chen, Division of Gastroenterology, Union Hospital, Tongji Medical College, Huazhong University of Science and Technology, 1277 Jiefang Avenue, Wuhan, Hubei 430022, P.R. China

E-mail: jiechen06@yahoo.com

Key words: vagotomy, gastric motility, gastric electrical stimulation, enteric glial cell, glial fibrillary acid protein, S100calcium binding protein B mechanism of the effect remains elusive, but maybe associated with activation of EGCs.

\section{Introduction}

Damage to the central nervous system, including brain injury and spinal cord injury, is common in the clinic. Traumatic brain injury (TBI) remains a public health problem and patients aged $\geq 75$ years tend to have a highest rate of TBI-associated deaths in the United States (1). Furthermore, traumatic brain injury and spinal cord injury may result in gastrointestinal dysfunction. Head injury predominantly delays gastric emptying in patients with short injury duration $(2,3)$. Similarly, spinal cord injury is known to contribute to prolong gastric emptying of solid meals (4).

The gastrointestinal tract is involved in double innervation: To the central nervous system and the enteric nervous system (ENS). The effects of the central nervous system are transmitted to the ENS predominantly via cholinergic fibers in the vagus nerve. Vagal activities are positively associated with gastric emptying, and electroacupuncture alleviates the delayed gastric emptying through a vagal mechanism (5). In addition, hyperglycemia damages vagal afferents, which inhibits gastric motility, while hyperglycemia activates the vagal efferent pathway to modulate gastric relaxation (6). Therefore, increasing attention has been dedicated to the potential mechanisms underlying gastrointestinal dysmotility resulting from the injury of central nervous system.

The ENS is the system that dominates the gastrointestinal activities independently by the central nervous system (7). The ENS is comprised of neurons and glial cells which are distributed in the myenteric and the submucosal plexus (8). Enteric glial cells (EGCs) are more abundant than enteric neurons (9) and are tightly packed around enteric neurons. Injured EGC lose functions of neuronal maintenance and survival and have been linked with ENS abnormalities (10). EGCs have an influence on mast cells, which participate in obstructed defecation (11). Previous studies have indicated that the reduced EGCs attenuate $\mathrm{Ca}^{2+}$-mediated responses through connexin-43 causing gastrointestinal motility disorder (12). 
The digestive tract loses control of the central nervous system following vagotomy, which is partly analogous to brain injury or spinal cord injury (13). The effects of vagotomy on gastric motility and EGCs are unknown. Whether SGES improves the gastric motility disorder and restore the change of EGCs resulting from vagotomy remains elusive. SGES may be able to alleviate gastrointestinal dysfunction. Therefore, the aims of the present study were to investigate the changes of gastric emptying and EGCs in the stomach with a course of subdiaphragmatic vagotomy in rats prior to and following SGES for further study on the underlying mechanism of the effect of EGCs in gastrointestinal motility.

\section{Materials and methods}

Animals. A total of 52 adult male Sprague Dawley rats (weighing 250-350 g) were used in the study. They were housed under normal laboratory conditions of $22^{\circ} \mathrm{C}$ and $12 \mathrm{~h}$ dark/light cycle, and were given food and water ad libitum. All animal protocols were approved by the Animals Care and Use Committee of Huazhong University of Science and Technology (Wuhan, China). The rats were obtained from the Experimental Center of Tongji Medical College (Wuhan, China). They were randomly divided into two groups: The control group, which involved incisions of the abdomen; and the subdiaphragmatic vagotomy group. These groups were further divided into subgroups. The control group consisted of early control group (ECN, 7 days, $\mathrm{n}=6$ ) and terminal control group (TCN, 56 days, $n=6)$. The experimental group contained the early subdiaphragmatic vagotomy group (ESDV, 7 days, $n=20)$ and the terminal subdiaphragmatic vagotomy group (TSDV, 56 days, $n=20$ ). Finally, the ESDV and TSDV were divided into three groups, which were either treated with sham gastric electrical stimulation $(n=6)$, short-term SGES (30 min/day, 7 days, $n=7$ ) or long-term SGES (30 $\mathrm{min} /$ day, 21 days, $\mathrm{n}=7$ ), respectively.

Surgical procedure. Following fasting the subdiaphragmatic vagotomized rats for $24 \mathrm{~h}$, the rats were anesthetized with an intraperitoneal injection of $1 \%$ pentobarbital sodium $(40 \mathrm{mg} / \mathrm{kg}$; Boster Biological Technology, Ltd., Wuhan, China). Under aseptic conditions, the stomach and subdiaphragmatic esophagus were exposed. Ventral and dorsal subdiaphagmatic vagi were cut completely, in addition to surrounding mesenteries. Simultaneously, a pair of temporary cardiac pacing wires (Medtronic, Dublin, Ireland) was placed on the serosal surface of the stomach. The pair was mounted in the middle of the greater curvature for stimulation. The implanted stimulating electrodes travelled separately from the subcutaneous layer of the backside to the top of the head. Penicillin (Boster Biological Technology, Ltd.) was used locally to prevent infection. The muscular layer was stimulated following one week of recovery.

SGES. SGES was performed as previously described by Yang et al (14). Briefly, each stimulation consisted of a long pulse (300 msec, $4 \mathrm{~mA}$ ) followed with five short pulses (0.33 msec, $100 \mathrm{~Hz}, 4 \mathrm{~mA})$. The stimulation was synchronized with the peak of gastric intrinsic slow waves.

Gastric emptying test. The authors used a modified gastric emptying model, as described previously (15). Phenol red
$(0.5 \mathrm{mg} / \mathrm{ml})$ and carboxymethylcellulose $(15 \mathrm{mg} / \mathrm{ml})$ were thoroughly mixed as test meal. At the scheduled time, the rats were fasted for $24 \mathrm{~h}$ before $2 \mathrm{ml}$ phenol red solution was administered to their stomachs. The stomachs were removed with the gastroesophageal junction and the pylorus harvested after $30 \mathrm{~min}$. Gastric content was rinsed in physiological saline up to $20 \mathrm{ml}$; then $20 \mathrm{ml} \mathrm{NaOH}(0.5 \mathrm{M})$ was added. The solution was mixed and allowed to stand for $1 \mathrm{~h}$ at room temperature. Following this, $5 \mathrm{ml}$ supernatant was removed and placed in a centrifuge tube with $0.5 \mathrm{ml}$ trichloroacetic acid $(20 \%$, w/v). Centrifugation $\left(1,050 \mathrm{x} \mathrm{g}, 4^{\circ} \mathrm{C}, 10 \mathrm{~min}\right)$ separated the phenol red solution (supernatant) from solution containing $5 \mathrm{ml}$ supernatant and $0.5 \mathrm{ml}$ trichloroacetic acid. The absorption value of phenol red was identified using a spectrophotometer at $560 \mathrm{~nm}$. Meanwhile, $2 \mathrm{ml}$ phenol red solution, $18 \mathrm{ml}$ physiological saline, $20 \mathrm{ml} \mathrm{NaOH}(0.5 \mathrm{~mol} / \mathrm{l})$ and $4 \mathrm{ml}$ trichloroacetic acid $(20 \%, w / v)$ represented a standard sample. Absorption value of the standard sample was measured as above. The gastric emptying rate was obtained by: 1-(phenol red absorption value from the stomach of animals sacrificed $30 \mathrm{~min}$ after the test meal/standard sample absorption value).

$R N A$ extraction and reverse transcription-quantitative polymerase chain reaction $(R T-q P C R)$. RT-qPCR was used to measure the expression levels of the $\mathrm{S} 100 \mathrm{~B} / \mathrm{glial}$ fibrillary acidic protein (GFAP) gene. RNA was isolated from stomach tissue using TRIzol reagent (Invitrogen; Thermo Fisher Scientific, Inc.). Single-stranded cDNA was synthesized with PrimeScript ${ }^{\mathrm{TM}}$ RT Master Mix (Takara Biotechnology Co., Ltd., Dalian, China). Primer sequences were as follows: S100B, forward 5'-GAGCAGGAAGTGGTGGACAAA-3' and reverse 5'-CACTCCCCATCCCCATCTT-3'; GFAP, forward 5'-TGACCGCTTTGCTAGCTACATC-3' and reverse 5'-GCGCCTTGTTTTGCTGTTC-3'; and GAPDH, forward 5'-GTATGACTCTACCCACGGCAAGT-3' and reverse 5'-TTCCCGTTGATGACCAGCTT-3'. GAPDH acted as an internal control. A total of $10 \mu \mathrm{l}$ PCR reaction volume was used, and included: $0.5 \mu \mathrm{l}$ upstream primer (Invitrogen; Thermo Fisher, Scientific, Inc.), $0.5 \mu 1$ downstream primer (Invitrogen; Thermo Fisher Scientific, Inc.), $5 \mu 1 \mathrm{SYBR}-$ Green

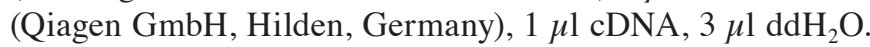
All PCR reactions following the established procedure were quantified using the ABI-OneStep real-time system (Applied Biosystems; Thermo Fisher Scientific, Inc.). Gene relative expression levels were calculated with the $2^{-\Delta \Delta \mathrm{Ct}}$ method (16).

Double-labeling immunofluorescence. Gastric tissues were double-labeled for enteric glial markers, S100B and GFAP protein. The acquired tissues were fixed in $4 \%$ paraformaldehyde for 6-24 hand cut into small segments of $\sim 5 \mathrm{~mm}$. The segments were then embedded into paraffin in a vacuum and sliced into at a thickness of $5 \mu \mathrm{m}$. Sections were boiled 1-2 min in citrate buffer for antigen retrieval after dewaxed in xylene and hydrated in the graded ethanol solutions. Following this, nonspecific binding sites were blocked by $5 \%$ bovine serum albumin (Boster Biological Technology, Ltd.) for $30 \mathrm{~min}$ at room temperature. The sections were then incubated with the following primary antibodies: Rabbit anti-S100B (1:300; cat. no. sc-136061; Santa Cruz Biotechnology, Inc., Dallas, 
TX, USA) and goat anti-GFAP (1:300; cat. no. ab53554; Abcam, Cambridge, MA, USA). Each section was incubated at $4^{\circ} \mathrm{C}$ overnight. Sections were washed 3 times in PBS following re-warming to room temperature for $60 \mathrm{~min}$ the following day. Sections were then incubated with secondary antibodies for Alexa Fluor 488-donkey anti-rabbit IgG (1:100 dilution; AntGene Biotech Co., Ltd, Wuhan, China) and AlexaFluor 594-donkey anti-goat IgG (1:200 dilution; AntGene Biotech Co., Ltd.) and kept in the dark at $37^{\circ} \mathrm{C}$ for 90 min. Following washing 3 times in PBS, sections were treated with Hoechst stain $(1: 1,500)$ at $37^{\circ} \mathrm{C}$ for $10 \mathrm{~min}$. Sections were sealed with fluorescence quenching agent (Boster Biological Technology, Ltd.) and observed using laser scanning confocal microscopy (Nikon Corporation, Tokyo, Japan). Image analysis was performed using Image Pro5.1 (Media Cybernetics, Inc., Rockville, MD, USA). Quantification was reported as mean signal per field, from 6 random fields per sample.

Transmission electron microscopy. The specimens of the antrum were fixed in $2.5 \%$ glutaraldehyde $(\mathrm{pH} 7.4)$ for $2 \mathrm{~h}$ at $4^{\circ} \mathrm{C}$ and rinsed with $0.1 \mathrm{M}$ PBS twice and immersed in $1 \% \mathrm{O}_{\mathrm{s}} \mathrm{O}_{4}$ $(\mathrm{pH}$ 7.4) for $1 \mathrm{~h}$. Then they were dehydrated with graded alcohol, embedded in Epon (Boster Biological Technology, Ltd.), cut into ultra-thin sections with an ultramicrotome (Leica Microsystems GmbH, Wetzlar, Germany), stained with lead citrate for $10 \mathrm{~min}$. Finally, these sections were viewed using a transmission electron microscope (Tecnai G2 12, FEI; Thermo Fisher Scientific, Inc.).

Statistical analysis. The mean value was used for statistical analysis and the results were presented as mean \pm standard error. One-way analysis of variance, followed by Bonferroni post hoc test, was performed to evaluate the difference between normal control groups and the subgroups of subdiaphragamtic vagotomy. $\mathrm{P}<0.05$ was considered to indicate a statistically significant difference. All calculations were performed using SPSS software (version 17.0; SPSS, Inc., Chicago, IL, USA).

\section{Results}

Assessment of gastric motility. Gastric emptying rates in different subdiaphragmatic vagotomy groups are presented in Fig. 1. In the early groups, ESDV demonstrated a faster gastric emptying when compared with the ECN group $(\mathrm{P}<0.05)$. In the terminal groups, gastric emptying was decreased in TSDV, when compared with TCN $(\mathrm{P}<0.05)$ and long-term SGES significantly improved delayed gastric emptying compared with TSDV $(\mathrm{P}<0.05)$. In addition, long-term SGES was more effective in accelerating delayed gastric emptying than short-term SGES $(\mathrm{P}<0.05)$.

Analysis of S10OB and GFAP mRNA expression. The mRNA expression of S100B and GFAP was measured by RT-qPCR (Fig. 2). During the course of subdiaphragmatic vagotomy, expression levels of S100B and GFAP were decreased gradually. However, mRNA expression of S100B and GFAP decreased significantly in the TSDV group compared with the TCN group $(\mathrm{P}<0.05)$. In the terminal groups, long-term

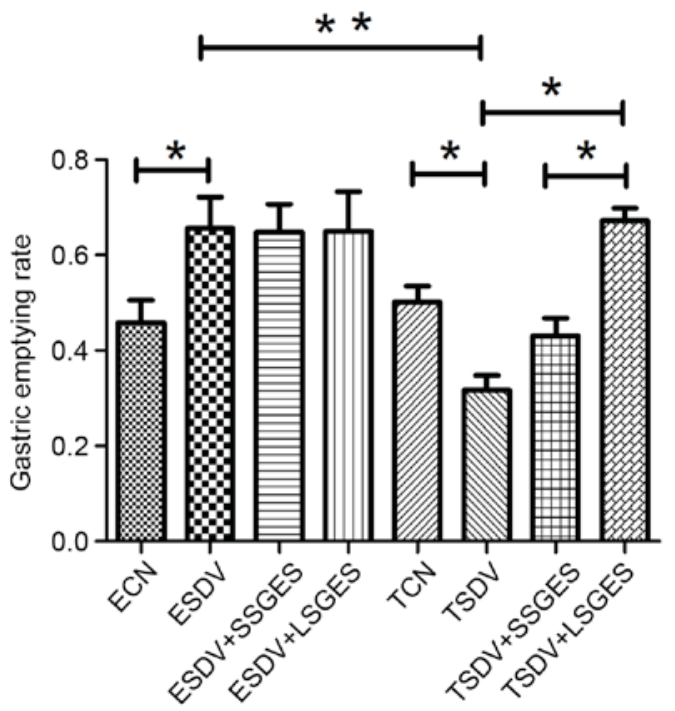

Figure 1. Gastric emptying rates in different experimental groups. ${ }^{*} \mathrm{P}<0.05$ and ${ }^{* *} \mathrm{P}<0.01$, with comparisons indicated by lines. ECN, early control; TCN, terminal control; ESDV, early subdiaphragmantic vagotomy; TSDV, terminal subdiaphragmatic vagotomy; SSGES, short-term synchronized gastric electrical stimulation of dual pulse; LSGES, long-term synchronized gastric electrical stimulation of dual pulse.

SGES increased mRNA expression of S100B and GFAP and expression levels were higher compared with short-term SGES $(\mathrm{P}<0.05)$.

Assessment of double-labeling immunofluorescence. S100B and GFAP proteins were evaluated by double-labeling immunofluorescence (Fig. 3A). There was no significant change between the early groups ( $\mathrm{P}>0.05$; Fig. 3B). S100B and GFAP protein expression was decreased gradually in the course of subdiaphragmatic vagotomy (Figs. 3B). The protein expression decreased significantly in the TSDV group, when compared with those of the TCN group $(\mathrm{P}<0.05$; Fig. $3 \mathrm{~B})$. Long-term SGES significantly upregulated S100B and GFAP protein expression $(\mathrm{P}<0.05)$ and was more effective at $\mathrm{S} 100 \mathrm{~B}$ and GFAP than short-term SGES. ( $\mathrm{P}<0.05$; Figs. 3B).

Assessment of ultrastructure of EGCs. The ultrastructure of EGC in the myenteric plexus was demonstrated by transmission electron microscopy (Fig. 4). There were abundant cell organelles containing mitochondria, filaments and the smooth and rough endoplasmic reticulum in the cytoplast of EGCs in the ECN and TCN groups (Fig. 4). The damaged ultrastructural features involved swelling of mitochondria, dilation of the endoplasmic reticulum, decreased numbers of filaments and condensed chromatin, and were identified in the ESDV and TSDV groups (Fig. 4) and the latter was marked than the former. In the short-term or long-term SGES, the numbers of mitochondria and filaments increased (Fig. 4). Unexpectedly, an apoptotic enteric neuron was observed in the TSDV group (Fig. 4).

\section{Discussion}

The results of the present study clearly demonstrated that EGCs were severely damaged and gastric emptying was delayed 

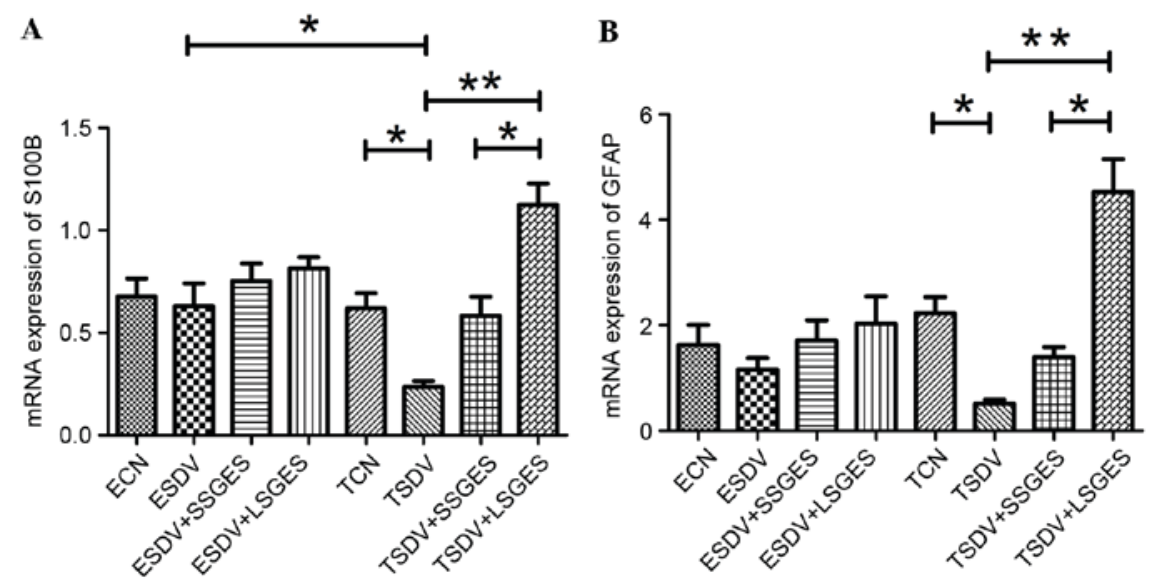

Figure 2. The mRNA expression of S100B and GFAP in response to SGES. mRNA expression levels of (A) S100B and (B) GFAP were measured by reverse transcription-quantitative polymerase chain reaction in the different experimental groups. ${ }^{*} \mathrm{P}<0.05$ and ${ }^{* *} \mathrm{P}<0.01$, with comparisons indicated by lines. S100B, calcium binding protein A1; GFAP, glial fibrillary acidic protein; SGES, synchronized dual pulse gastric electrical stimulation; ESDV, early subdiaphragmantic vagotomy; TSDV, terminal subdiaphragmatic vagotomy; TCN, terminal control; LSGES, long-term synchronized gastric electrical stimulation of dual pulse; SSGES, short-term synchronized gastric electrical stimulation of dual pulse.

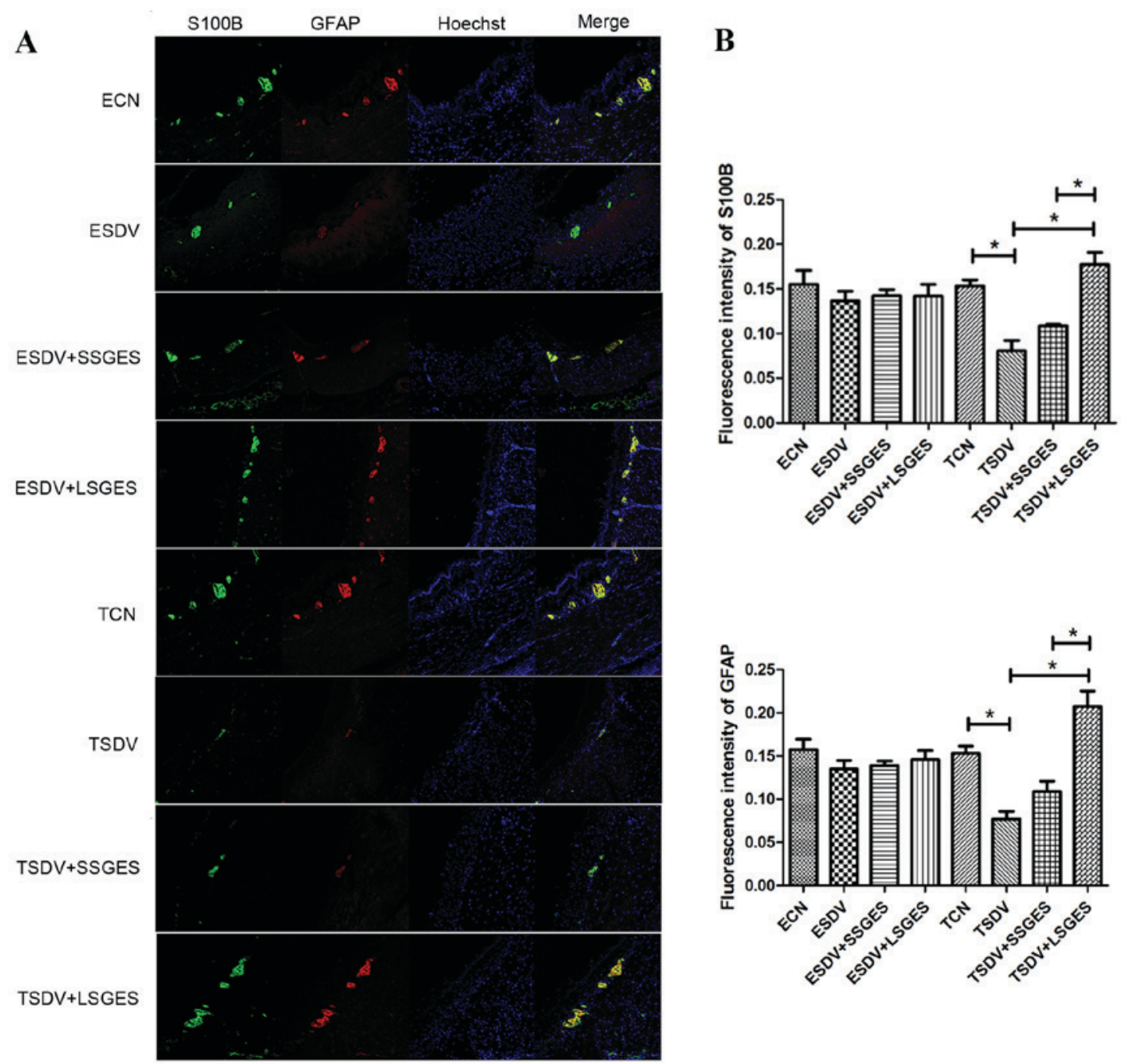

Figure 3. Double-labeling with S100B (green) and GFAP (red) in the myenteric plexus of the stomach in the different experimental groups. Nuclei were stained with Hoechst (blue). (A) Representative images from each experimental group. (B) Quantification of relative fluorescence intensity for S100B and GFAP per experimental group presented as mean \pm standard error of the mean. "P $<0.05$, with comparisons indicated by lines. S100B, calcium binding protein; GFAP, glial fibrillary acidic protein; ECN, early control; ESDV, early subdiaphragmantic vagotomy; SSGES, short-term synchronized gastric electrical stimulation of dual pulse; LSGES, long-term synchronized gastric electrical stimulation of dual pulse; TCN, terminal control; TSDV, terminal subdiaphragmatic vagotomy. 

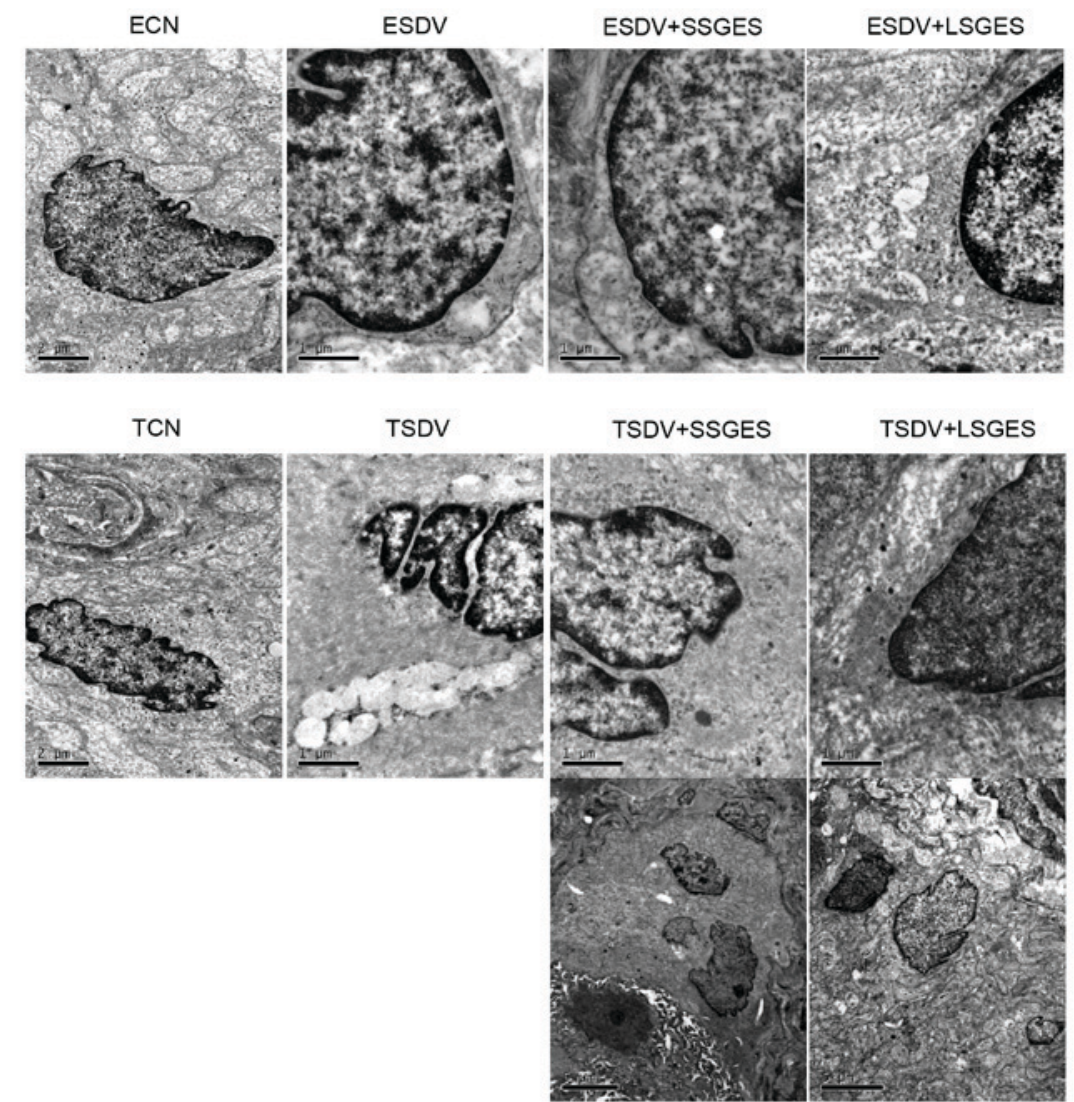

Figure 4. Ultrastructural alterations of EGCs in the myenteric plexus. Specimens of the antrum in the different experimental groups were analyzed by transmission electron microscopy. EGCs, enteric glial cells; ECN, early control; TCN, terminal control; ESDV, early subdiaphragmantic vagotomy; TSDV, terminal subdiaphragmatic vagotomy; SSGES, short-term synchronized gastric electrical stimulation of dual pulse; LSGES, long-term synchronized gastric electrical stimulation of dual pulse.

following subdiaphragmatic vagotomy in rats. Meanwhile, the data indicate that SGES may activate injured EGCs and improve gastric motility. In addition, EGCs had a certain reserve capacity in the early subdiaphragmatic vagotomy for the expression of S100B and GFAP mostly the same amount compared with the early control group.

Vagal activities have clear influence on gastrointestinal motility. Vagal innervation regulates the contractile pattern and contractile activity of stomach (17) and vagal nerve stimuli promotes activation of EGCs in a burn-induced intestinal injury mouse model (18). In the current study, gastric emptying was delayed in the TSDV group, while it remained unchanged in the ESDV group. The underlying mechanism may be that the EGCs initiated a compensatory function modulating gastric motility in the ESDV group and beyond its ability in the TSDV group. Therefore, SGES improved the symptoms of the vagotomy-induced delayed gastric motility, which is consistent with previous literature $(7,14)$. To the best of the authors' knowledge, the vagal fibers primarily promoted gastrointestinal movement and secretion, while sympathetic fibers have the opposite effect. There are conflicting results concerning which one has the main role in the ENS. A previous paper reported that only sympathetic fibers released ATP to activate EGCs, and then EGCs selectively responded to sympathetic activities in the guinea pig distal colon (19). The possible explanation for the discordance may be that sympathetic fibers and vagal fibers innervate the specific regions of gastrointestinal tract respectively and different species may also affect the results.

EGCs have been previously reported to respond to pro-inflammatory stimuli by producing nitric oxide or increasing GFAP+ enteric glia (20). In addition, glia cell lined-derived neurotrophic factor (GDNF) has been demonstrated to promote the survival of enteric neurons, and the decrease of GDNF may be involved in the damage to enteric neurons in diabetics (21). Glial-derived s-nitrosoglutathione restored the function of the mucosal barrier through upregulating F-actin and tight junction associated proteins (22). EGCs act as glutamatergic neurotransmitter receptors, and expression of glial metabotropic glutamate receptor subtype 5 is decreased during colitis (23). EGCs have been associated with the modulation of the intestinal epithelial barrier in acute intestinal ischemia reperfusion injury, leading to activation of EGCs as barrier protection (24). In addition, nicotinic cholinergic agonists promote EGC activation, and the activated EGCs modulate intestinal barrier integrity by inhibiting the nuclear factor- $\kappa \mathrm{B}$ pathway $(22,25)$. EGCs serve an indispensable role in maintaining the intestinal epithelial barrier and participate in the inflammatory response. Therefore, activation of EGCs by different stimulating factors is necessary. In the present study, EGCs were damaged seriously in the TSDV group, but SGES activated EGCs. Nevertheless, how the activated EGCs regulate gastrointestinal barrier function in subdiaphragamtic vagotomy is yet to be investigated. 
EGCs participate in regulating gastrointestinal motility. The John Cunningham (JC) virus, a polymavirus, can affect glial cells in the brain, resulting in fatal diseases. In a previous study, EGCs in the myenteric plexus were demonstrated to be infected with the JC virus in chronic idiopathic intestinal pseudo-obstruction, which may be associated with the lesion of intestinal propulsive motility (26). However, an additional study indicated that mucosa-associated glial cell networks decline in diabetics caused by a high-fat diet, while myenteric glial cells saw no change in the early and the late disease periods (27). A previous study demonstrated that EGCs in the myenteric plexus of the stomach were decreased in terminal diabetic rats (14). EGCs, together with enteric ganglion cells and interstitial cells of Cajal, were significantly decreased in idiopathic slow transit constipated patients (28).

EGCs communicate with enteric neurons dynamically which has been recognized increasingly important. Neuronal activity may be detected by EGCs through neurotransmitters (29). Likewise, EGCs may be activated by specific neurotransmitters or receptors. Mechanical stimulation and ATP activate EGCs, resulting in the increasing of intracellular calcium levels; these increases propagate to neighboring cells partly via gap junctions (30). Gulbransen and Sharkey (31) reported that EGCs express $\mathrm{P} 2 \mathrm{Y} 4$ receptors, which predominantly mediate ATP detection. In addition, EGCs respond to serotonergic and cholinergic signaling (32). In the presented subdiaphragmatic vagotomy rat model, it was verified that EGCs were activated by SGES. However, the reasons underlying the alterations to $\mathrm{Ca}^{2+}$ levels, together with $\mathrm{K}^{+}$and $\mathrm{Na}^{+}$in activated EGCs, remain unclear. There are certain difficulties to overcome, for example, how to acquire viable EGCs sufficiently. In the future, more studies should be conducted on ion changes in EGCs.

$\mathrm{S} 100 \mathrm{~B}$ is a $\mathrm{Ca}^{2+}$ binding protein, which acts as a marker of EGC activation. The expression of S100B can have a trophic or toxic effect in EGCs, depending on its extracellular concentration (33). Pathogens cause a dramatic upregulation of S100B expression and NO products in human-derived EGCs in a host-bacteria interaction model (34). This indicates that glial-derived S100B is involved in the pro-inflammatory response, which agrees with previous research $(35,36)$. The present study demonstrated the activation of EGCs through S100B gene/protein upregulation, which was involved in improving gastric emptying.

EGCs contain a dense intermediate filament and GFAP, which is another marker for EGCs in the gut. The distortion of EGCs was associated with the alterations to the position and form of GFAP; the distorted EGCs then induced damage to neighboring enteric neurons (37). Furthermore, GFAP was upregulated and dephosphorylated in the EGCs of patients with Parkinson's disease (38). The activation of EGCs led to GFAP overexpression in the gastrointestinal tract; the same result as the present finding. Based on the expression of GFAP, von Boyen et al (20) divided EGCs into two different types: GFAP- and GFAP+. The significantly increased GFAP+ EGCs respond to pro-inflammatory cytokines, which participate in modulating the integrity of the inflamed gut.

In conclusion, the authors displayed that SGES was concerned with increased activation of EGCs and promoted gastric motility in rats with subdiaphragmatic vagotomy.
However, further evidence is required to indicate that how EGCs communicate with enteric neurons and with interstitial cells of Cajal and smooth muscle cells to regulate the gastrointestinal functions. Finally, the ultimate aim is to make EGCs accessible as a potential therapeutic target point in the diseases of enteric neuropathy.

\section{Acknowledgements}

The current study was supported by a grant from the National Natural Science Foundation of China (grant no. 81170342).

\section{References}

1. Coronado VG, Xu L, Basavaraju SV, McGuire LC, Wald MM, Faul MD, Guzman BR and Hemphill JD; Centers for Disease Control and Prevention (CDC): Surveillance for Traumatic Brain Injury-Deaths-United States, 1997-2007. MMWR Surveill Summ 60: 1-32, 2011.

2. Kao CH, ChangLai SP, Chieng PU and Yen TC: Gastric emptying in head-injured patients. Am J Gastroenterol 93: 1108-1112, 1998.

3. Pinto TF, Rocha R, Paula CA and de Jesus RP: Tolerance to enteral nutrition therapy in traumatic brain injury patients. Brain Inj 26: 1113-1117, 2012.

4. Fynne L, Worsoe J, Gregersen T, Schlageter V, Laurberg S and Krogh K: Gastric and small intestinal dysfunction in spinal cord injury patients. Acta Neurol Scand 125: 123-138, 2012.

5. Song J, Yin J, Sallam HS, Bai T, Chen Y and Chen JD: Electroacupuncture improves burn-induced impairment in gastric motility mediated via the vagal mechanism in rats. Neurogastroenterol Motil 25: 807-e635, 2013.

6. Zhou SY, Lu YX and Owyang C: Gastric relaxation induced by hyperglycemia is mediated by vagal afferent pathways in the rat. Am J Physiol Gastrointest Liver Physiol 294: G1158-G1164, 2008.

7. Chen J, Koothan T and Chen JD: Synchronized gastric electrical stimulation improves vagotomy-induced impairment in gastric accommodation via the nitrergic pathway in dogs. Am J Physiol Gastrointest Liver Physiol 296: G310-G318, 2009.

8. Furness JB: The Enteric Nervous System. Blackwell Publishing, Oxford, p274, 2006

9. Gulbransen BD: Enteric glia. Morgan \& Claypool Publishers, San Rafael, CA, 2014.

10. De Giorgio R, Giancola F, Boschetti E, Abdo H, Lardeux B and Neunlist M: Enteric glia and neuroprotection: Basic and clinical aspects. Am J Physiol Gastrointest Liver Physiol 303: G887-G893, 2012.

11. Bassotti G, Villanacci V, Nascimbeni R, Cadei M, Manenti S, Antonelli E, Fanini L and Salerni B: Increase of colonic mast cells in obstructed defecation and their relationship with enteric glia. Dig Dis Sci 57: 65-71, 2012.

12. McClain JL, Grubišić V, Fried D, Gomez-Suarez RA, Leinninger GM, Sévigny J, Parpura V and Gulbransen BD: Ca2+ responses in enteric glia are mediated by connexin- 43 hemichannels and modulate colonic transit in mice. Gastroenterol 146: 497-507.e1, 2014

13. Collares EF and Vinagre AM: Evidence of the effect of dipyrone on the central nervous system as a determinant of delayed gastric emptying observed in rats after its administration. Braz J Med Biol Res 36: 1375-1382, 2003.

14. Yang W, Wang N, Shi X and Chen J: Synchronized dual pulse gastric electrical stimulation induces activation of enteric glial cells in rats with diabetic gastroparesis. Gastroenterol Res Pract 2014: 964071, 2014.

15. Di Marzo V, Capasso R, Matias I, Aviello G, Petrosino S, Borrelli F, Romano B, Orlando P, Capasso F and Izzo AA: The role of endocannabinoids in the regulation of gastric emptying: Alterations in mice fed a high-fat diet. Br J Pharmacol 153: 1272-1280, 2008.

16. Livak KJ and Schmittgen TD: Analysis of relative gene expression data using real-time quantitative PCR and the 2(-Delta Delta C(T)) method. Methods 25: 402-408, 2001.

17. Tanaka T, Kendrick ML, Zyromski NJ, Meile T and Sarr MG: Vagal innervation modulates motor pattern but not initiation of canine gastric migrating motor complex. Am J Physiol Gastrointest Liver Physiol 281: G283-G292, 2001. 
18. Costantini TW, Bansal V,Krzyzaniak M,Putnam JG,Peterson CY, Loomis WH, Wolf P, Baird A, Eliceiri BP and Coimbra R: Vagal nerve stimulation protects against burn-induced intestinal injury through activation of enteric glia cells. Am J Physiol Gastrointest Liver Physiol 299: G1308-G1318, 2010.

19. Gulbransen BD, Bains JS and Sharkey KA: Enteric glia are targets of the sympathetic innervation of the myenteric plexus in the guinea pig distal colon. J Neurosci 30: 6801-6809, 2010.

20. von Boyen GB, Steinkamp M, Reinshagen M, Schäfer KH, Adler G and Kirsch J: Proinflammatory cytokines increase glial fibrillary acidic protein expression in enteric glia. Gut 53: 222-228, 2004

21. Du F, Wang L, Qian W and Liu S: Loss of enteric neurons accompanied by decreased expression of GDNF and PI3K/Akt pathway in diabetic rats. Neurogastroenterol Motil 21: 1229-e114, 2009.

22. Savidge TC, Newman P, Pothoulakis C, Ruhl A, Neunlist M, Bourreille A, Hurst R and Sofroniew MV: Enteric glia regulate intestinal barrier function and inflammation via release of S-nitrosoglutathione. Gastroenterol 132: 1344-1358, 2007.

23. Nasser Y, Keenan CM, Ma AC, McCafferty DM and Sharkey KA: Expression of a functional metabotropic glutamate receptor 5 on enteric glia is altered in states of inflammation. Glia 55: 859-872, 2007.

24. Xiao W, Wang W, Chen W, Sun L, Li X, Zhang C and Yang H: GDNF is involved in the barrier-inducing effect of enteric glial cells on intestinal epithelial cells under acute ischemia reperfusion stimulation. Mol Neurobiol 50: 274-289, 2014.

25. Cheadle GA, Costantini TW, Bansal V, Eliceiri BP and Coimbra R: Cholinergic signaling in the gut: A novel mechanism of barrier protection through activation of enteric glia cells. Surg Infect (Larchmt) 15: 387-393, 2014.

26. Selgrad M, De Giorgio R, Fini L, Cogliandro RF, Williams S, Stanghellini V, Barbara G, Tonini M, Corinaldesi R, Genta RM, et al: JC virus infects the enteric glia of patients with chronic idiopathic intestinal pseudo-obstruction. Gut 58: 25-32, 2009.

27. Stenkamp-Strahm C, Patterson S, Boren J, Gericke M and Balemba O: High-fat diet and age-dependent effects on enteric glial cell populations of mouse small intestine. Auton Neurosci 177: 199-210, 2013.

28. Bassotti G, Villanacci V, Maurer CA, Fisogni S, Di Fabio F, Cadei M, Morelli A, Panagiotis T, Cathomas G and Salerni B: The role of glial cells and apoptosis of enteric neurones in the neuropathology of intractable slow transit constipation. Gut 55 41-46, 2006.
29. Gulbransen BD, Bains JS and Sharkey KA: Enteric glia are targets of the sympathetic innervation of the myenteric plexus in the guinea pig distal colon. J Neurosci 30: 6801-6809, 2010.

30. Gomes P, Chevalier J, Boesmans W, Roosen L, van den Abbeel V, Neunlist M, Tack J and Vanden Berghe P: ATP-dependent paracrine communication between enteric neurons and glia in a primary cell culture derived from embryonic mice. Neurogastroenterol Motil 21: 870-e62, 2009.

31. Gulbransen BD and Sharkey KA: Purinergic neuron-to-glia signaling in the enteric nervous system. Gastroenterol 136: 1349-1358, 2009.

32. Boesmans W, Cirillo C, Van den Abbeel V, Van den Haute C, Depoortere I, Tack J and Vanden Berghe P: Neurotransmitters involved in fast excitatory neurotransmission directly activate enteric glial cells. Neurogastroenterol Motil 25: e151-e160, 2013.

33. Cirillo C, Sarnelli G, Esposito G, Turco F, Steardo L and Cuomo R: S100B protein in the gut: The evidence for enteroglial-sustained intestinal inflammation. World J Gastroenterol 17: 1261-1266, 2011.

34. Turco F, Sarnelli G, Cirillo C, Palumbo I, De Giorgi F, D'Alessandro A, Cammarota M, Giuliano M and Cuomo R: Enteroglial-derived S100B protein integrates bacteria-induced Toll-like receptor signalling in human enteric glial cells. Gut 63: 105-115, 2014.

35. Cirillo C, Sarnelli G, Turco F, Mango A, Grosso M, Aprea G, Masone S and Cuomo R: Proinflammatory stimuli activates human-derived enteroglial cells and induces autocrine nitric oxide production. Neurogastroenterol Motil 23: e372-e382, 2011.

36. Esposito G, Cirillo C, Sarnelli G, De Filippis D, D'Armiento FP, Rocco A, Nardone G, Petruzzelli R, Grosso M, Izzo P, et al: Enteric glial-derived S100B protein stimulates nitric oxide production in celiac disease. Gastroenterol 133: 918-925, 2007.

37. Thacker M, Rivera LR, Cho HJ and Furness JB: The relationship between glial distortion and neuronal changes following intestinal ischemia and reperfusion. Neurogastroenterol Motil 23: e500-e509, 2011.

38. Clairembault $\mathrm{T}$, Kamphuis W, Leclair-Visonneau L, Rolli-Derkinderen M, Coron E, Neunlist M, Hol EM and Derkinderen P: Enteric GFAP expression and phosphorylation in Parkinson's disease. J Neurochem 130: 805-815, 2014. 